\title{
An industry update: what is the latest news in therapeutic delivery?
}

This Industry Update covers the period from 1 through 30 April 2017, and is based on information sourced from company press releases, scientific literature, patents and various news websites. Biogen expanded its portfolio of developmental drugs targeting neurological diseases by licensing a drug from Bristol-Myers Squibb which is currently under investigation for the treatment of progressive supranuclear palsy. Takeda announced a deal with NuBiyota focused on developing treatments for gastrointestinal disorders, based on the manipulation of microbes living in the gut. Novartis announced an expansion of its neuroscience collaboration with Amgen as well as licensing of a developmental compound to treat dry eye. EmulateBio announced a collaboration with the US FDA to explore and evaluate its organs-on-chip technology in toxicological testing of food products, but which has wider opportunities in drug development. In the treatment of pulmonary hypertension, Vectura announced the commercial launch of its Fox nebulizer technology to deliver a Bayer drug and Respira announced a deal in which it exclusively licensed an inhaled drug-device product to United Therapeutics. Published research suggested that the benefit of pumps over multiple injections in the treatment of diabetes maybe more due to training than technology, and a group from the University of Pennsylvania showed that electrical stimulation of the brain can improve memory function but only when applied at the right time. Alzheon published work that explains the mechanism behind its developmental Alzheimer's drug.

First draft submitted: 26 May 2017; Accepted for publication: 1 June 2017; Published online: 21 July 2017

Keywords: combination products • emerging technologies • parenteral delivery

\section{Business development}

\section{Lyndra, Inc.}

Lyndra, Inc. (MA, USA) announced that it has raised US\$23M in a Series A round of financing.

Lyndra is one of a long list of companies to be established out of Professor Bob Langer's laboratory at MIT and is focused on developing a novel therapeutic oral delivery platform that allows drugs to be formulated to have much longer therapeutic lifetimes than conventional formulations allow. It is argued that less frequent administration should improve adherence and that it can also maintain a more constant in vivo concentration of the drug which can maintain efficacy as well as reduce drug side effects.

The technology which was developed at MIT with support from Bill and Melinda Gates Foundation, uses gelatin encapsulation to protect and maintain the drug in the gut until the dose has been released after which the capsule is broken down into smaller pieces that can then pass out via the gastrointestinal (GI) tract.

The funding was led by Polaris Partners, with participation from several other partners lain Simpson

Ixico Plc - 4th Floor Griffin Court 15 Long Lane London EC1A 9PN, UK Author for correspondence: Tel.: +44 07717496237 iain.simpson123@gmail.com 
including Quark Venture and GF Securities, Yonghua Capital, Healthlink Capital, Partners Healthcare and Suffolk Equity [1].

\section{Licensing \& collaboration}

\section{Biogen \& Bristol-Myers Squibb}

Biogen (NASDAQ: BIIB; MA, USA) announced that it has licensed an anti-tau monoclonal antibody (mAbs) from Bristol-Myers Squibb (NYSE: BMY; NY, USA). BMS-986168 is currently in a Phase II study for progressive supranuclear palsy, a rare disease which has some similarity to Parkinson's disease. However, the drug has potential applications for other neurodegenerative diseases, most notably Alzheimer's disease (AD), for which Biogen already has three drugs in development, aducanumab and BAN2401 which are both $\mathrm{mAbs}$ that target amyloid plaques, and E2609, a BACE inhibitor, which stops the formation of amyloid. So, the addition of BMS-986168 gives it access to another treatment mechanism; targeting $\mathrm{T}$ proteins that form into neurofibrillary tangles that accumulate in the brain and are suspected of causing synapse death. Many industry experts see anti-tau therapies as a highly promising approach to treating $\mathrm{AD}$, especially given the recent failures of several promising antiamyloid drugs.

BMS acquired BMS-986168 through its acquisition of iPierian in 2014, claiming at the time it was an opportunity to diversify and move into the treatment of neurodegenerative disease which had not been a recent focus for the business. The deal with Biogen comes just a month after the company announced the appointment of leasing oncologist Dr Thomas Lynch, to the role of $\mathrm{CSO}$, which might signal a focusing of BMS's programs on oncology, a well-established area of strength for the business.

Biogen will pay $\$ 300 \mathrm{M}$ to BMS up front plus royalties of up to $\$ 410 \mathrm{M}$ as well as taking on BMS's obligations to iPierian's shareholders [2].

\section{Takeda \& NuBiyota}

Takeda Pharmaceutical Company Limited (TSE:4502; Osaka, Japan) announced a strategic collaboration with biotech start-up NuBiyota (NJ, USA) to develop microbiome-based products to treat GI disorders, a major area of therapeutic focus for Takeda. Although there is limited public information on NuBiyota, it was founded based on work conducted at Dr Emma AllenVercoe's laboratory at the University of Guelph in Canada. Dr Allen-Vercoe's has developed a world-leading capability to grow microbes in controlled conditions and NuBiyota was established to transition this capability into therapeutic products. The collaboration will look at both microbial consortia (where groups of cells live symbiotically) and microbiome-derived bioactives to investigate how the microbiome can be utilized for treatment of GI diseases.

Takeda has a long history in the treatment of GI disorders and has recently increased its interest in microbiome and recently signed other microbiome-related deals notably earlier with month with Finch Therapeutics (MA, USA) and in early 2016 with Enterome (Paris, France). Takeda has also signed several deals in GI disease, including in March 2017, a research collaboration with University of Chicago and the Icahn School of Medicine at Mount Sinai in New York, focusing on inflammatory bowel disease research and care. The collaboration includes the development of disease markers and the use of a digital platform that monitors real-time disease status and gathers information on disease factors for each patient, offering the potential of more personalized treatment regimens.

Terms of the deal between Takeda and NuBiyota were not released but the latter will receive an upfront and for success-based development payments based on regulatory and commercial milestones as well as royalties from sales of the developed therapeutics [3].

\section{Novartis \& Amgen}

Novartis (VTX: NOVN; Basel, Switzerland) announced that it has expanded its neuroscience collaboration with Amgen (AMGN:NASDAQ; CA, USA) to cover the commercialization of erenumab (AMG 334), an investigational drug for the prevention of migraine. Novartis will gain marketing rights for the drug in Canada as well as maintaining those it has elsewhere in the world other than in Japan where Amgen keeps the rights. The two companies have entered into a co-commercialization agreement for the USA where Novartis's established neuroscience presence should help rapidly grow sales once the drug is approved following filing in the USA which is still anticipated in the first half of 2017.

Erenumab targets and seeks to block the CGRP receptor, believed to play a critical role in mediating migraine pain. Results published last year from a Phase III study showed that the drug reduced the average number of days of headache for study participants on the drug from 8 days to around 3.5 days compared with 1.8 days for participants on placebo.

The development of anti-CGRP $\mathrm{mAbs}$ is likely to be a competitive arena over the coming years with Lilly targeting filing in 2017 for galcanezumab. Teva (TLV: TEVA; Petah Tikva, Israel) and Alder (NASDAQ:ALDR, WA, USA), are also anticipated to follow with their regulatory submissions for fremanezumab and eptinezumab, respectively in the next year. 
A variety of factors are likely to affect success including dosing regimen: patients are considered to prefer less frequent administration, especially when this involves injection. Alder has stated that they are targeting dosing four-times per year by infusion, which is unlikely to be suitable for self-administration at home, although they are also exploring other formulations for self-administration. The other companies are looking at fortnightly or monthly subcutaneous injections that could be done at home by self-administration using an autoinjector, the increasingly common approach for other already marketed mAbs.

Novartis and Amgen entered into a global collaboration in August 2015 jointly to develop and commercialize neuroscience therapeutics. In addition to programs targeting migraine, the collaboration also includes two Alzheimer's drugs in late clinical development as well as further preclinical compounds [4].

\section{EmulateBio}

EmulateBio (MA, USA) announced a collaboration with the US FDA to explore and evaluate its organson-chip technology in toxicological testing of food products - including foods, dietary supplements and cosmetics. Although this collaboration is not focused on developing therapeutics, the decision by the FDA to explore its potential in regulatory testing is potentially significant. EmulateBio already has several pharmarelated deals in place including with the Michael $\mathrm{J}$ Fox Foundation to identify new drug candidates for the treatment of Parkinson's Disease, with Merck to look at inflammatory diseases, Covance (a leading clinical research organization) to explore applications in preclinical testing of new drugs and with Seres Therapeutics to look at microbiome-based therapeutics in inflammatory bowel disease and other serious conditions.

The technology platform is based on combining microengineering with living human cells to create what EmulateBio term an Organ-Chip, a living, microengineered environment recreating the natural physiology and mechanical forces that cells experience in various organs including the lung, kidney, intestine and liver. The microengineered structures, which are about the size of an AA battery contain tiny hollow channels lined with tens of thousands of living human cells and tissues [5].

\section{Novartis}

Novartis announced that it has exercised an option to in-license a drug ECF843, from Lubris LLC (MA, USA), which is being developed to treat dry eye, a condition that affects over $344 \mathrm{M}$ people globally. ECF843, is a recombinant form of human lubricin, an endogenous human protein found in humans and expressed in areas of high shear stress and friction including the tear film in the eye where it binds to and protects tissues of the ocular surface.

Lubricin has been observed to be deficient in sufferers of dry eye and Phase II studies conducted by Lubris and reported late last year, showed a 70\% reduction in several key symptoms from baseline, including foreign body sensation, burning/stinging, pain, sticky feeling, blurred vision and photophobia in at least one eye.

The drug has the potential to be a first-in-class $\mathrm{Rx}$ treatment in dry eye, which is considered a condition with high unmet need.

Novartis has a long-standing franchise in ophthalmic treatments spanning both over-the-counter products (OTC), such as lubricating eye drops, as well as prescription products, such as Lucentis, used to treat more serious conditions such as wet macular degeneration.

Lubris continues to explore other opportunities for lubricin which include use as a coating on contact lenses and in treating osteoarthritis [6].

\section{Respira}

Respira Therapeutics (NM, USA) announced a strategic collaboration with United Therapeutics Corporation (NASDAQ: UTHR; MD, USA) for the development and exclusive license of an inhaled drug-device product for the treatment of pulmonary hypertension $(\mathrm{PH})$.

The company has been developing novel dry powder inhaler technologies, which it claims can deliver dry powder drug products to the lung more effectively than other dry powder inhalers. The technology uses the patient's breath to drive an oscillating sphere which breaks up the drug to create respirable particles that are then delivered to the lung. Both single-use and multidose versions are under development and although the technology could be applicable more widely to inhaled drugs, the company has focused on $\mathrm{PH}$ with three programs initiated using an investigational drug RT234.

With this deal, United Therapeutics will become the lead investor in a Series B funding, providing Respira with development funding to advance RT234 through the FDA approval. Previous investors in the business also participated in the new finding round.

$\mathrm{PH}$ is a rare but serious and progressive disease which if untreated has a poor life expectancy. United Therapeutics is a leading player in its treatment but faces competition from other entrants such as Steadymed (NASDAQ:STDY; CA, USA) which is developing a convenient wearable pump to deliver a version of treprostinil, another of United Therapeutics drugs as well as 
existing players such as Bayer, mentioned elsewhere in this industry review, who have just launched their drug in a new more convenient nebulizer system [7].

\section{Regulatory news \& approvals}

\section{Merck \& Co}

Merck \& Co, announced that it has received a complete response letter form the FDA relating to its supplemental new drug applications for JANUVIA ${ }^{\circledR}$ (sitagliptin) and JANUMET ${ }^{\circledR}$ (sitagliptin and metfor$\mathrm{min}$ ) which are prescribed to improve glycemic control in adults with Type 2 diabetes mellitus. The company had been seeking a label change to include data from the recent TECOS study that shows the drugs do not increase cardiovascular risk.

The label update would have help Merck in competing both with in-class DPP- 4 drugs notably Onglyza from AstraZeneca and Nesina from Takeda, for which there is evidence of increased risk of heart failure and also other out-of-class antidiabetic drugs, notably Jardiance ${ }^{\circledR}$ (empagliflozin), marketed by Lilly which is an SGLT2 inhibitor. Late last year, the FDA approved a new indication for Jardiance to reduce the risk of cardiovascular death in adults with Type 2 diabetes and established cardiovascular disease, which Lilly claims is a first oral Type 2 diabetes medication. Type 2 diabetes is known to substantially increase the lifetime risk of heart failure so antidiabetic drugs that increase the risk of the latter are a concern and medications that can address both conditions have a distinct advantage.

Merck is continuing to develop its own SGLT2 inhibitor.

Merck said that it was reviewing the complete response letter will then discuss next steps with the FDA [8].

\section{Market news}

\section{Vectura Plc}

Vectura (LSE:VEC.L; Chippenham, UK) announced the first commercial launch of its novel Fox nebulizer for the delivery Bayer's drug Ventavis which is used to treat pulmonary arterial hypertension.

The FOX ${ }^{\circledR}$ device is a small handheld, battery-powered device that uses a vibrating mesh technology to nebulize liquid drug into a fine respirable mist which is delivered to the lung by inhalation by the patient. The Breelib device used to deliver Ventavis is a modified version of the FOX technology developed as a collaboration between Vectura and Bayer. Vectura acquired FOX when it purchased the German company Activaero in 2014.
Ventavis is already marketed with another nebulizers, the I-Neb AAD from Philips and the VENTANEB from NEBU-TEC. Dosing is six- to nine-times per day with each treatment taking 4-10 min, but with Breelib, this can be reduced to around $3 \mathrm{~min}$, which improves convenience.

Pulmonary arterial hypertension is a progressive and life-threatening disease in which constriction of the pulmonary arteries results in increased blood pressure which can lead to right heart failure and death. It has untreated median survival of $2-3$ years from time of diagnosis, but this is increasing as new treatment comes to the market.

The launch of Breelib has been launched initially in Poland but this will extend to other countries in the EU following approval of the device in late 2016 [9].

\section{Pumps no better than pens}

Research published in the British Medical Journal and reported by Reuters suggests that patients with Type 1 diabetes treated with insulin using pumps manage their blood sugar levels no better than those who use multiple injections, provided they get the same level training.

In the study, 260 patients at eight sites in the UK were enrolled onto an intensive training program which aims to teach participants how to manage flexibly their insulin administration, taking into account their diets.

Patients were then randomly assigned either a pump or multiple daily injections and monitored over a 2-year period. All participants initially had high HbAlc levels (average value: 9.1), indicative of poor glycemic control. At the end of the study periods, both groups showed that on average they had reduced HbAlc levels, but these were still above national guidelines. The pump group showed slightly more lowering but after correcting the results for site and patient effects, the difference was not seen to be significant. Patients using the pump on average reported higher satisfaction levels due to the relative ease-of-use benefits over multiple injections.

It is estimated that around $6 \%$ of UK adults with Type 1 diabetes use pumps, which is lower than in many comparable countries; the USA has one of the highest uptake rates at around $40 \%$. This difference in uptake is probably driven by cost as well as reimbursement rates - pumps cost around $£ 2500$ each plus a further $£ 1500$ a year for consumables (such as cannulas, batteries and dose chambers), which is significantly higher than the cost for injection devices. As a result, NICE guidance for the UK only supports their use in children and for adults who have failed to achieve good control using multiple injections. Several studies using 
pumps have shown good improvements in glycemic control, supporting the argument that pumps should be more widely offered in the UK. However, this new research suggests that the better results seen in other pump studies looking might be better explained by the level of training provided in these studies rather than by the technology itself [10-12]. Lack of training is a source of nonadherence in other disease areas where self-administration of medication using delivery devices is used. For example, in the treatment of respiratory disease using inhaled drugs, patient misuse rates often exceed $50 \%$ and even healthcare professionals often do not know the correct usage procedure. This is driver for the use of smartphone apps and intelligent devices and trainer devices to teach users and then to reinforce correct use [13].

\section{Patents, novel technology \& science}

\section{Chronic fatigue syndrome linked with differences in gut bacteria}

Research from a group at Columbia University (NY, USA) published in the journal Microbiome, suggests that chronic fatigue syndrome (CFS) might be linked with imbalances in bacteria in the gut.

CFS is a disorder in which people present extreme and long-term fatigue that is neither the result of another medical condition nor improved by rest. Estimates of prevalence vary, in part as the disorder is hard to isolate from other conditions but is estimated to be in the range of between 7 and 300 cases per 100,000 adults.

In the research, shotgun metagenomic sequencing of fecal samples was used to evaluate bacterial diversity and measure the abundance of microbes from a cohort of 50 subjects with CFS and 50 healthy controls. Results showed differences in microbiome count and type between the cohorts. Furthermore, with the bacterial analysis, it was possible to identify between subjects who also suffered from irritable bowel syndrome (IBS), a common co-morbidity with CFS.

The authors suggest that analysis of gut bacteria along with measurements of plasma cells and metabolic pathways might enable better diagnostic and the development of better therapies to treat the condition [14,15].

\section{Electrical stimulation of the brain can improve memory function}

A team of scientists at the University of Pennsylvania has shown that electrical stimulation of the brain can improve memory function when applied at a time when memory failure is predicted to occur. Notably, the opposite effect occurs if the stimulation is done at times when memory is effective.
Working with neurosurgical patients receiving treatment for epilepsy, the team first used machine learning (a process in which algorithms that can learn from and make predictions based on analysis of the data) to analyze signals from various parts of the brain in order to establish high and low points in memory function. Processing these data allows identification biomarkers of effective memory function and patterns of activity that were associated with the effective creation of new memories.

The effectiveness of the electrostimulation was assessed using a series of memory tests in which the participants were asked to study and recall lists of common words while receiving stimulation to areas of the brain identified to be associated with memory encoding. Stimulation was given at both high- and low-functioning brain states and results showed positive effects at times when the brain was showing poor function and the opposite when it was functioning well.

Previous studies on deep brain stimulation have had variable results. This new research offers new insight into the process suggesting that the timing of the stimulation is critical.

The research is part of the 'Restoring Active Memory' a 4-year DARPA project funded by the Department of Defense with the goal of developing a fully implantable device that will restore lost memory function. The emphasis is on treating traumatic brain injury, a serious cause of disability affecting an estimated 1.7 million people in the USA [16].

\section{Identification of therapeutic mechanism for tramiprosate in reduction of amyloid $\beta$ oligomers}

Alzheon (MA, USA) reported results that show the therapeutic method of action for tramiprosate, the active ingredient in their developmental drug ALZ801.

The formation of amyloid beta $(A \beta)$ oligomers has been recognized as a key process in the development and progression of $\mathrm{AD}$ for around 40 years. However, it has proved challenging to develop drugs that target this process safe and effectively, and there have been a number of high-profile drug failures in late-stage trials in recent years. The team from Alzheon used a combination of ion mobility spectrometry-mass spectrometry (IMS-MS), NMR and molecular modeling/dynamic simulation to show that tramiprosate binds with three key amino acid side chains on the $A \beta$ monomer forming a stable structure that inhibits the formation of the harmful insoluble oligomer. The concentration of tramiprosate achieved in the brain at an effective dose could be determined from previous 
pharmacokinetic data, and further analysis then showed this would be sufficient to achieve complete prevention of $A \beta$ oligomer formation.

Tramiprosate was the first antiamyloid drug to be taken into Phase III around 10 years ago, although, the studies conducted by Canadian company Neurochem failed to show a statistically significant effect over placebo for the three primary study end points. One particular challenge was the high level of variability in results from different sites that hindered the statistical analysis which may have been due to difficulty in getting consistency in the study measures or possibly the effect of other medications that the subjects were still using. But there was some evidence from MRI scans that the treated group showed a reduction in hippocampal volume loss (which has been linked with the progression of dementia) compared with the placebo group. Furthermore, Phase II studies had shown lowering of $A \beta$ levels in the brain, as measured in cerebral spinal fluid.

Alzheon recognized the potential of tramiprosate and acquired the rights in 2013 from BELLUS Health,

\section{References}

1 Lyndra's Long-Acting Drug Delivery Technology lands \$23M investment.

www.xconomy.com/boston/2017/04/13/lyndras-long-actingdrug-delivery-technology-lands-23m-investment

2 Biogen licenses Phase II anti-tau antibody from Bristol-Myers Squibb.

http://media.biogen.com/press-release/corporate/biogenlicenses-phase-2-anti-tau-antibody-bristol-myers-squibb

3 Takeda enters into strategic collaboration with NuBiyota for Microbiome Therapeutics.

www.takeda.com/newsroom/newsreleases/2017/takedaenters-into-strategic-collaboration-with-nubiyota-formicrobiome-therapeutics/

4 Novartis expands global collaboration with Amgen to commercialize first-in-class AMG 334 (erenumab) program in migraine prevention in the US and Canada.

https://www.novartis.com/news/media-releases/novartisexpands-global-collaboration-amgen-commercialize-firstclass-amg-334

5 FDA signs collaborative agreement with Emulate, Inc. to use organs-on-chips technology as a toxicology testing platform for understanding how products affect human health and safety. https://emulatebio.com/press/fda-collab-agreement-emulate

6 Novartis to strengthen R\&D pipeline by in-licensing ECF843 for ophthalmic indications. www.novartis.com/news/media-releases/novartis-strengthenrd-pipeline-licensing-ecf843-ophthalmic-indications

7 Respira Therapeutics announces collaboration with United Therapeutics to treat pulmonary hypertension. www.prnewswire.com/news-releases/respira-therapeutics-
Inc (the successor company to Neurochem) but also chose to adopt a precision medicine approach to future development. They focused on patients with the APOE4 gene, which is known to increase the risk of $\mathrm{AD}$ as well as resulting in a more aggressive form of the disease. They also chose to deliver tramiprosate as a prodrug in order to improve the tolerability, pharmacokinetic and pharmacodynamic profile and metabolic stability of the drug.

Alzheon plans to accelerate the development of ALZ-801 and take it into a Phase III study in the near future [17].

\section{Financial \& competing interests disclosure}

The author has no relevant affiliations or financial involvement with any organization or entity with a financial interest in or financial conflict with the subject matter or materials discussed in the manuscript. This includes employment, consultancies, honoraria, stock ownership or options, expert testimony, grants or patents received or pending, or royalties.

No writing assistance was utilized in the production of this manuscript. announces-collaboration-with-united-therapeutics-to-treatpulmonary-hypertension-300438430.html

8 FDA shoots down Merck's bid to add lack of heart risks to Januvia's label.

www.fiercepharma.com/pharma/fda-shoots-down-merck-sbid-to-add-lack-heart-risks-to-januvia-s-label

9 First launch of a product incorporating Vectura’s FOX ${ }^{\circledR}$ smart nebuliser technology.

http://www.vectura.com/news/first-launch-productincorporating-vecturas-fox-smart-nebuliser-technology/

10 With same training, insulin pumps no better than injections. http://uk.reuters.com/article/us-health-insulin-patienteducation-idUKKBN1772JG

11 REPOSE Study Group. Relative effectiveness of insulin pump treatment over multiple daily injections and structured education during flexible intensive insulin treatment for type 1 diabetes: cluster randomised trial (REPOSE). BMJ. doi:10.1136/bmj.j1285 (2017) (Epub ahead of print). www.bmj.com/content/356/bmj.j1285

12 Continuous subcutaneous insulin infusion for the treatment of diabetes mellitus. www.nice.org.uk/guidance/TA151/chapter/1-Guidance

13 Inhaler Error Steering Committee Inhaler competence in asthma: common errors, barriers to use and recommended solutions. Respir. Med. 107(1), 37-46 (2013). www.sciencedirect.com/science/article/pii/ S0954611112003587

14 Chronic fatigue syndrome linked with differences in gut bacteria.

www.livescience.com/58859-chronic-fatigue-syndrome-gutbacteria-ibs.html

15 Nagy-Szakal D, Williams BL, Mishra N et al. 
Fecal metagenomic profiles in subgroups of patients with myalgic encephalomyelitis/chronic fatigue syndrome. Microbiome 5(1), 44 (2017). www.ncbi.nlm.nih.gov/pubmed/28441964 during lapses.

https://news.upenn.edu/news/penn-researchers-show-brainstimulation-restores-memory-during-lapses

17 Alzheon scientists discover novel therapeutic mechanism of inhibition of formation of toxic beta amyloid oligomers, key driver of Alzheimer's disease pathogenesis.

http://alzheon.com/alzheon-scientists-discover-noveltherapeutic-mechanism-inhibition-formation-toxic-betaamyloid-oligomers-key-driver-alzheimers-disease-pathogenesis 
